\title{
Radio occultation techniques for probing the ionosphere
}

\author{
NORBERT JAKOWSKI $\left({ }^{1}\right)$, REINHART LEITINGER $\left({ }^{2}\right)$ and MATTHEW ANGLING $\left({ }^{3}\right)$ \\ $\left(^{1}\right)$ Deutsches Zentrum für Luft und Raumfahrt (DLR), Institut für Kommunikation und Navigation, \\ Neustrelitz, Germany \\ $\left(^{2}\right)$ Institute of Physics, Institute for Geophysics, Astrophysics and Meteorology (IGAM), \\ University of Graz, Austria \\ $\left(^{3}\right)$ Centre for RF Propagation and Atmospheric Research, QinetiQ, Malvern, U.K.
}

GPS radio occultation measurements establish the basis for a new remote sensing technique for vertical profile information on the electron density of the entire ionosphere from satellite orbit heights down to the bottomside. No other profiling technique such as vertical sounding or incoherent scatter, unifies vertical profiling through the entire ionosphere with global coverage. Inversion methods are described both for vertical profiling as well as for tree dimensional electron density reconstructions of the ionosphere. In three dimensional electron density reconstructions using signals from Global Navigation Satellite Systems (GNSS), the Ionospheric Radio Occultation (IRO) measurements provide vertical information which is complementary to the information obtained by ground based measurements. Assessment of achievable accuracy and spatial resolution are addressed by simulation studies. IRO measurements have been carried out onboard the German CHAMP satellite since 11 April 2001 on a routine basis. Assuming a spherically layered ionosphere, up to about 150 Electron Density Profiles (EDPs) per day are retrieved within a latency of $3 \mathrm{~h}$. Validation results obtained by using independent data sources are reported. The validation with vertical sounding data in mid-latitudes indicates a small positive bias in the plasma frequency of up to about $0.5 \mathrm{MHz}$ throughout the entire profile. Averages of the numerous EDPs show wellknown ionospheric phenomena such as the equatorial anomaly, the winter anomaly and the expansion of the profile with increasing solar energy input. It is concluded that CHAMP-IRO measurements have the potential to establish global data sets of vertical electron density profiles for developing and improving global ionospheric models and to provide operational space weather information.

\subsection{INTRODUCTION}

The GPS limb sounding technique has been demonstrated to be a powerful tool for remote sensing of the Earth's neutral atmosphere and ionosphere by the GPS/MET instrument, flown on the Microlab-1 Low Earth Orbiting (LEO) satellite (e.g., Kursinski et al., 1997; Hajj and Romans, 1998). LEO missions such as CHAMP and SAC-C that carry a dual frequency GPS receiver onboard, offer a unique chance for improving measuring techniques and algorithms for retrieving the electron density and, for improving our knowledge of ionospheric behaviour and monitoring the actual state of the global ionosphere on a continuous basis. 
Radio occultation measurements of GPS carrier phases onboard a LEO satellite like CHAMP enable the computation of the vertical refractivity profile from the LEO satellite orbit height down to the Earth's surface. Since the index of refractivity of the ionosphere depends mainly on the number of free electrons, the inversion of the measured signals can provide the vertical Electron Density Profile (EDP) (e.g., Jakowski et al., 2002a).

In the stratospheric and tropospheric altitude ranges, vertical neutral gas temperature or water vapour profiles may be derived from the refractivity profile (Wickert et al., 2002).

Combined ground and space based GNSS measurements provide an excellent basis for reconstructing the three dimensional structure of the electron density distribution by data assimilation (e.g., Angling and Cannon, 2004) or tomographic techniques including additional data sources (e.g., Mitchell and Spencer, 2002). Recent achievements in this field are described in Section 7.6.

The geo-science satellite mission CHAMP has provided high quality IRO data since 11 April 2001 on a routine basis. So far more than 80000 profiles have been retrieved from IRO measurements onboard CHAMP by using a model assisted retrieval technique that is briefly described in Section 7.3. However, before this new data type can be used for detailed ionospheric studies, the retrievals have to be validated in a systematic manner. Within the COST 271 Action a radio occultation validation group was established to support the validation of the IRO retrieved vertical EDPs by means of vertical sounding data from the following European vertical sounding stations: Athens, Dourbes, Juliusruh, Rome and Tortosa (Jakowski et al., 2004b). Section 7.7 deals with the potential of IRO derived EDPs for ionospheric research, modelling and monitoring.

\subsection{Simulation STUdieS}

The inversion of Radio Occultation (RO) data belongs to the remote sounding methods and needs extensive assessment studies to prove its usefulness and to gain an impression on what accuracies can be achieved under which circumstances.

For an ideal geometry (transmitter and receiver remain in a plane and have identical orbital heights) there is a one to one relation between the inversion of RO data and the inversion of travel time data of seismic waves. The mathematically continuous inversion method for seismic waves, which was developed between 1906 and 1910 by Wiechert and Herglotz (Herglotz, 1907; Wiechert and Geiger, 1910; cited according to Jeffreys, 1976, and Bullen, 1963), assumes spherical geometry (the signal velocity depends on the distance $r$ from the centre of the Earth only) and gives unique results if the signal velocity increases or decreases monotonously with $r$. If we can assume straight line propagation of the GNSS signals this condition is not necessary and a continuous inversion of data with zero error would always give unique results. However, discretization causes errors which accumulate in the lower part of the inversion profile.

In principle, inversion of RO data is a very stable process. Even if the electron density distribution deviates strongly from spherical geometry inversion the inversion produces an electron density profile. However this profile is a «horizontally averaged» one which might contain height intervals with (meaningless) negative electron densities.

Apart from the effect of non-spherical electron density distributions, GPS to LEO occultation poses another problem: the transmitter is outside the plasmapause but the receiver is in the ionosphere. We have to eliminate the (slant) electron content of the plasmasphere and (with LEO heights below about $2000 \mathrm{~km}$ ) that of the upper ionosphere. Partially this is done by subtracting from all data the electron content measured for the upper end of the occultation (for the highest occultation ray perigee). The remaining influence of the upper ionosphere and that of the plasmasphere as well as the influence of «horizontal gradients» of electron density has to be assessed by means of model calculations.

Extensive calculations were carried out on the basis of the three dimensional and time dependent 
ionosphere/plasmasphere model NeUoG-plas (Hochegger and Leitinger, 2000). The following four parameters of electron density height profiles were investigated:

- peak height $h m F 2$;

- peak electron density $N m F 2$;

- topside scale height or topside slab thickness;

- a shape parameter for the bottomside ionosphere.

The assessment studies showed that in mid latitudes and, with some exceptions, in low latitudes, the most reliable parameter is peak height, followed by peak density and the shape of the topside ionosphere. Because of error accumulation the shape parameter for the bottomside ionosphere is substantially less reliable. The low latitudes exception region is the vicinity of one of the peaks of the equatorial anomaly and (to a lesser degree) the region around the dip equator.

It is clear that the geometry of the occultation plays an important role too. Excluding spread out occultations with projections of the ray perigees are distributed over regions with angular extensions $>5^{\circ}$, meridional cases (small longitude difference between GPS and LEO locations) give better results than zonal ones. With a LEO height around $800 \mathrm{~km}$ Hochegger and Leitinger (2000) found that for meridional occultations in mid latitudes the peak height error very rarely exceeds $30 \mathrm{~km}$ but remains $<20 \mathrm{~km}$ in more than $50 \%$ of the cases. For far more than $50 \%$ of the cases the peak density error was $<15 \%$.

A refined investigation brought proof for the assumption that the ground occultation point should not be at an electron content peak or in an electron content valley. The sharper the peak or valley, the less reliable are the inversion results. Of course, this is clearly due to the «component» of the horizontal electron density distribution that has even symmetry with respect to the ground occultation point. Model assisted inversion (Hochegger and Leitinger, 2001) was found to improve results substantially if enough data existed about the horizontal gradients of vertical electron content or peak electron density.

\subsection{IRO MEASUREMENTS ONBOARD CHAMP}

The German CHAMP (CHAllenging Minisatellite Payload) satellite was successfully launched into a near polar orbit $\left(I=87^{\circ}, h=450 \mathrm{~km}\right.$ ) by a Russian COSMOS rocket on 15 July 2000 (Reigber et al., 2000). The satellite is equipped with a dual frequency GPS receiver that enables the analysis of the $0.1 \mathrm{~Hz}$ sampled navigation data as well as GPS radio occultation measurements. Thus, the actual state of the ionosphere is permanently monitored near the CHAMP orbit plane.

The CHAMP science data are received at the DLR Remote Sensing Data Center Neustrelitz and then passed directly to the GeoForschungsZentrum (GFZ) Potsdam. The measured GPS data are automatically checked and preprocessed by the operational processing system (Wehrenpfennig et al., 2001). Due to the modular structure of the processing system high flexibility is achieved, and retrieval modules can be modified or replaced in the course of the CHAMP mission. The computed data products are submitted to the Information and Science Data Center (ISDC) of the GFZ Potsdam where they may accessed by the international science community.

The advanced «Black Jack» GPS receiver developed by the Jet Propulsion Laboratory (JPL) provides, on the one hand, precise time and orbit information. On the other hand, the receiver measures GPS carrier phases in the radio occultation or limb sounding mode starting at CHAMP orbit tangential heights down to the Earth surface with a sampling rate of $1 \mathrm{~Hz}$. Furthermore, the $0.1 \mathrm{~Hz}$ sampled navigation data may be used for reconstructing the topside ionosphere electron density distribution by data assimilation (Heise et al., 2002).

From about 200 IRO measurements about 150 Electron Density Profiles (EDPs) are successfully retrieved per day ( $c f$. fig. 7.1). Because the processing system works automatically, some EDP outliers cannot be avoided. The number of such 'unrealistic' profile outliers is in principal less than $1 \%$. All in all, more than 80000 profiles have been collected so far, forming a powerful database for more 


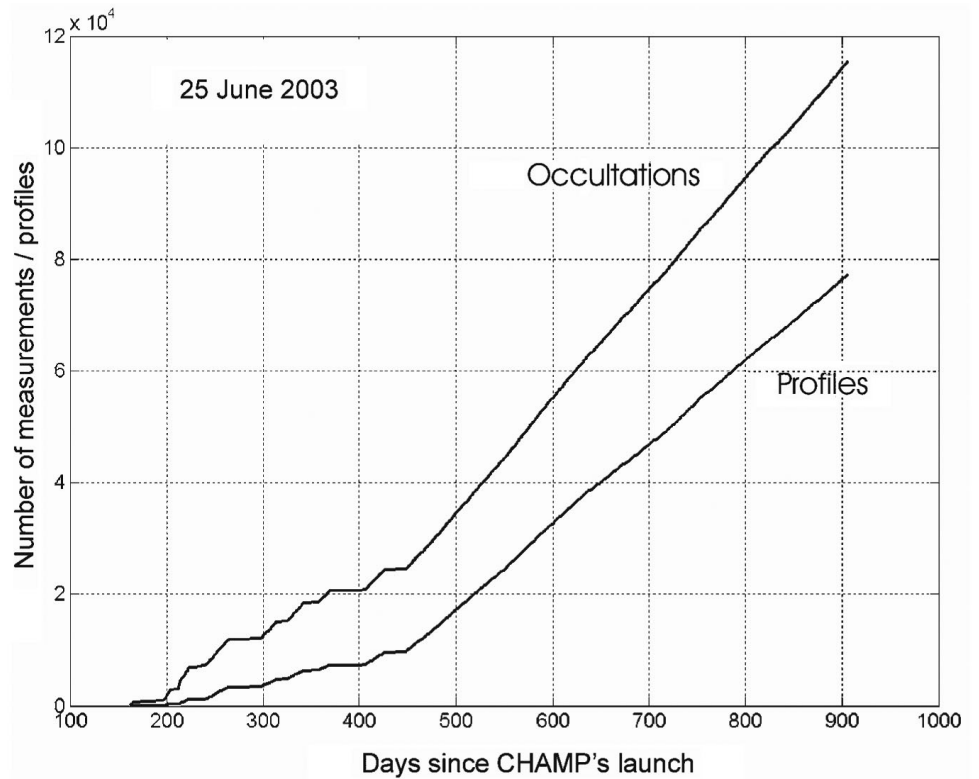

Fig. 7.1. Statistics of the number of measured GPS radio occultations on CHAMP and corresponding derived vertical electron density profiles.

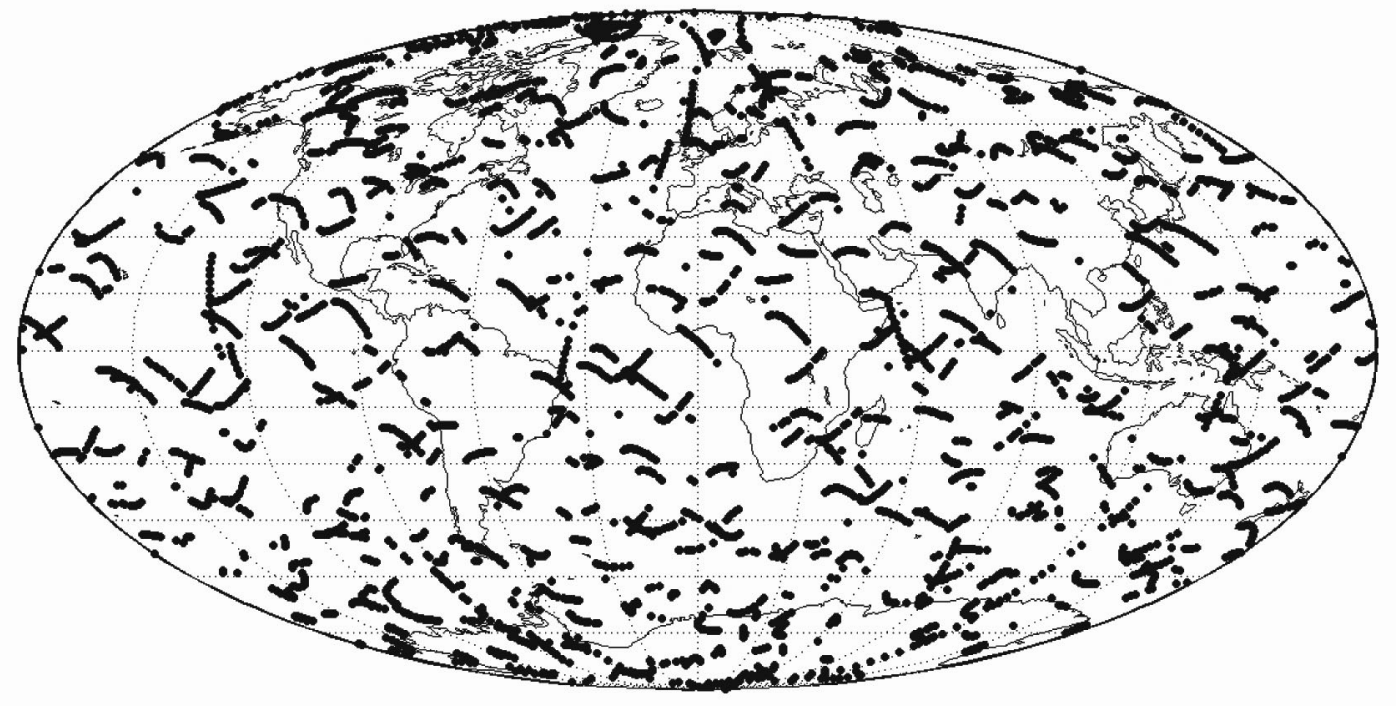

Fig. 7.2. Ionospheric radio occultation data coverage of CHAMP measurements during April 2002. 
detailed studies. As fig. 7.2 illustrates, due to the high inclination, the radio occultation measurements onboard CHAMP cover the global ionosphere. The radio occultation technique is a unique tool that enables the vertical profiling of the entire ionosphere from the topside down to the bottomside. Consequently, although there are some methodological constraints which are discussed in the following section, there is great potential for future applications.

To enable comparative studies with independent ionospheric measurements at least during special measuring campaigns, selected CHAMP data are stored at the TEC Evaluation Data Archive (TECEDA) that has been established within COST 271 activities at DLR Neustrelitz (cf. Stamper et al., 2004). Complete IRO data sets obtained onboard CHAMP may be obtained via the Information and Science Data Center (ISDC) Potsdam (http://isdc.gfzpotsdam.de/champ/welcome.html) or DLR directly. DLR routinely feeds the ISDC with ionospheric radio occultation data products.

\subsection{RETRIEVAl TECHNIQUe}

Since a number of ionospheric phenomena are accompanied by strong spatial plasma density gradients and furthermore, the path through the ionosphere is in the order of 1000-2000 km long, the spherical symmetry assumption of the Abel inversion technique does not, in general, hold. To overcome this methodological restriction, tomographic solutions are required. The tomographic approach developed for the CHAMP data analysis (Jakowski, 1999; Jakowski et al., 2002a), has the advantage that additional information (e.g., horizontal gradients) from ground based GPS measurements, models and/or other sources can easily be included in the reconstruction of the electron density profile, at least in post-processing.

Considering the GPS signal frequencies $L 1$ and $L 2$, the ionospheric phase delay $d_{I}$ may be estimated in a first-order approximation by

$$
d_{I}=K / f^{2} \int n_{e} d s \quad\left(\text { where } K=40.3 \mathrm{~m}^{3} \mathrm{~s}^{-2}\right)
$$

where $n_{e}$ is the electron density along the ray path $s$ (e.g., Davies, 1990). Due to the frequency dispersion the difference of $L 1$ and $L 2$ phases may be used to remove all other variable ranging parameters. Thus, according to eq. (7.1) the differential GPS phases provide the total electron content along the ray path through a spherically layered voxel structure (cf. fig. 7.3). The measured line integral TEC is here the sum of the product $n_{e} \times d s_{i}$ where $n_{e}$ is the mean electron density in voxel $i$ and $d s_{i}$ corresponds to the ray path length in voxel $i$ at measurement $j$. Simulations have shown that the ray path bending can be ignored in a first-order approximation (Schreiner et al., 1999). Hence, the ray path elements can be computed easily according to the satellite geometry defined by the positions of the transmitting GPS satellite and the LEO satellite where the signal is received. The electron density of different shells can successively be derived from a series of $1 \mathrm{~s}$ sampled measurements $j$ when the tangential point of occultation rays comes closer and closer to the Earth down to the bottom of the ionosphere ( $c f$. fig. 7.3).

If the satellite orbit is well above the F2-layer peak as in case of the Oerstedt and SAC-C satellite missions, the comparatively small plasmaspheric contribution can be considered to be a constant. This simplifying assumption cannot be applied to the CHAMP-IRO retrieval algorithms because CHAMP has a rather low orbit height of less than $450 \mathrm{~km}$, which will even further decrease with mission time. To overcome this upper boundary problem, a specific model assisted technique has been developed for the CHAMP data analysis (Jakowski, 1999; Jakowski et al., 2002a).

Practically, the solution starts with the first measurement at the greatest tangential height by using an adaptive model for the topside ionosphere and plasmasphere above the CHAMP orbit height. The adaptive model consists of a Chapman layer whose topside is extended by a slowly decaying exponential term with a scale height of $10000 \mathrm{~km}$. Crucial model parameters such as the plasma scale height at the upper boundary are determined by six iterations in order to ensure a smooth transition 


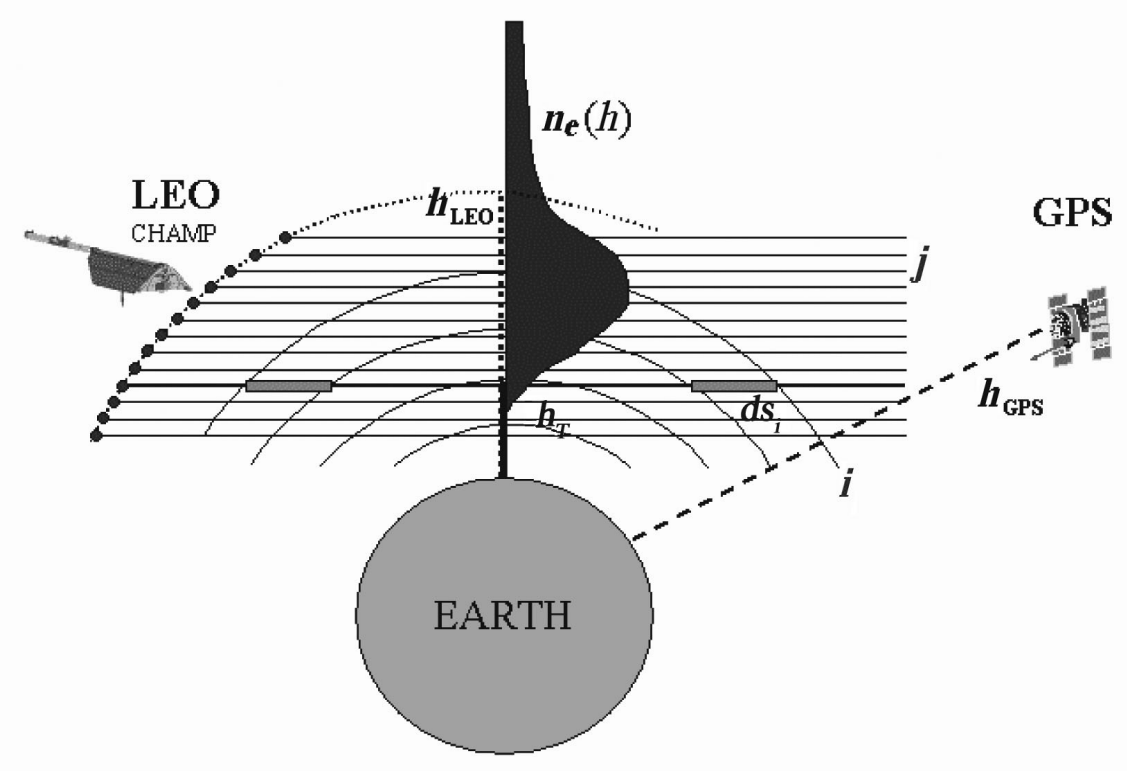

Fig. 7.3. Illustration of the retrieval technique used for CHAMP data analysis.

between model and measurements. It has been found that the most crucial element for improving the solution of the upper boundary problem is the topside scale height (Jakowski et al., 2002b). Recently attempts have been made to develop a topside scale height model that can be used as an initial guess for the retrieval procedure (Stankov and Jakowski, 2004).

To fulfil operational requirements, i.e. to come up with retrieval products within a latency of less than $3 \mathrm{~h}$, no further data are included in the retrieval procedure, i.e. for reasons of simplicity a spherically layered ionosphere is assumed. The retrieval can be improved if additional information, e.g., on horizontal gradients or local densities, is included in the retrieval procedure (e.g., Garcia Fernandez et al., 2003). Horizontal gradients can be deduced from horizontal TEC maps such as those produced from ground based GPS measurements in DLR/IKN since 1995 (http://www.kn.nz.dlr.de/).

\subsection{VALIDATION OF RO MEASUREMENTS FROM CHAMP}

Before any retrieved electron density profiles can be used in ionospheric research, the accuracy and reliability of the retrievals have to be estimated. This can be done by comparison with measurements obtained by quite different techniques such as vertical sounding, incoherent scatter radar or in situ measurements. Principally, the IRO derived electron density profiles provide a unique measure. The limb sounding technique provides profiles that describe the mean vertical electron density structure in comparable large areas with a diameter of up to about $3000 \mathrm{~km}$. Thus, a comparison with precise localised vertical sounding data should yield differences that are strongly biased by the local ionospheric structure.

The evaluation of IRO derived vertical EDPs from CHAMP was supported by a special validation group which has been established within the COST 271 activities. Subsequently, IRO retrieved elec- 
tron density profiles were compared with European vertical sounding (digisonde) data obtained in Juliusruh $\left(54.6^{\circ} \mathrm{N} ; 13.4^{\circ} \mathrm{E}\right)$, Athens $\left(38.0^{\circ} \mathrm{N} ; 23.5^{\circ} \mathrm{E}\right)$, Rome $\left(41.9^{\circ} \mathrm{N} ; 12.5^{\circ} \mathrm{E}\right)$, Tortosa $\left(40.8^{\circ} \mathrm{N}\right.$; $\left.0.5^{\circ} \mathrm{E}\right)$ and Dourbes $\left(50.1^{\circ} \mathrm{N} ; 4.6^{\circ} \mathrm{E}\right)$.

The results are shown in fig. 7.4 and indicate a systematic positive bias of the IRO data in the order of about $0.5 \mathrm{MHz}$ and a standard deviation from the mean of about $1 \mathrm{MHz}$ throughout the entire profile. Somewhat better results have been achieved with the Juliusruh station alone indicating that the validation process has to be continued (Jakowski et al., 2004b).

For validation the F2-layer electron peak density and height values the corresponding data were taken from globally distributed vertical sounding stations (SPIDR, O'Loughlin, 1997) and compared with IRO data within a cross section of about $1600 \mathrm{~km}$ diameter. The deviation distributions are based on 1006 and 509 values for $f 0 F 2$ and $h m F 2$, respectively. The absolute distribution function shifts by $0.18 \mathrm{MHz}$ for $f 0 F 2$ and $13.4 \mathrm{~km}$ for $h m F 2$. The RMS deviations of $1.28 \mathrm{MHz}$ and $46.8 \mathrm{~km}$ for $f 0 F 2$ and $h m F 2$, respectively, agree in principle with former estimations (e.g., Schreiner et al., 1999). The strong deviations in $h m F 2$ indicate that more comprehensive validation studies are needed.

Vertical electron density profiles throughout the ionosphere may be provided by incoherent scatter facilities. To study the high latitude ionosphere by different techniques, several CHAMP-EISCAT measuring campaigns were initiated in recent years such as the Satellite and Incoherent scatter Radar Cusp Studies (SIRCUS) campaign in 2001-2002. If incoherent scatter and IRO measurements coincide within a cross section diameter of about $1600 \mathrm{~km}$ and a time window of less than $30 \mathrm{~min}$, the derived EDPs yield valuable data sets for the evaluation of the IRO data. Within a special study (Stolle et al., 2004) 28 profiles measured under different geophysical conditions were analysed. It was found that the majority of profiles agree within the error ranges of the two methods. General-
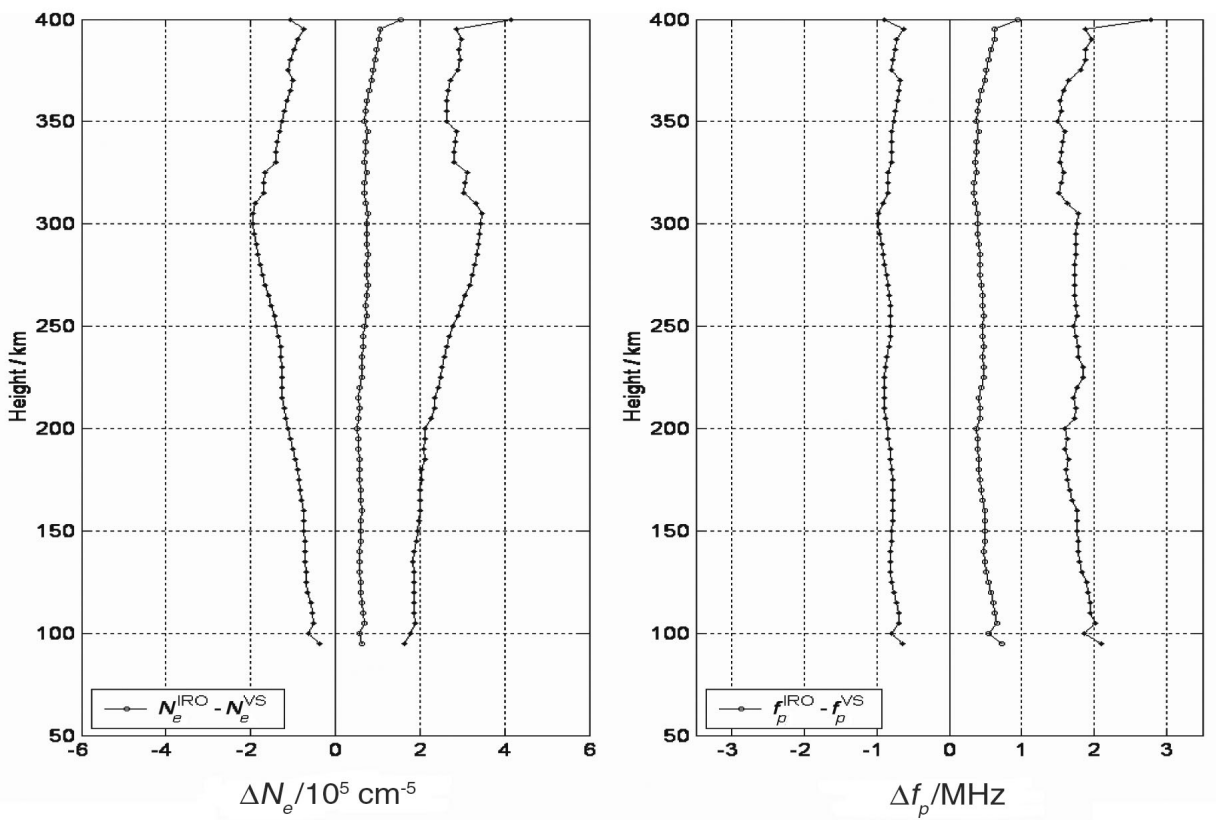

Fig. 7.4. Statistical comparison of IRO and coinciding vertical sounding derived EDPs (474 profiles, Athens, Dourbes, Juliusruh, Rome, Tortosa, 01/02-03/03). Left panel: electron density $N_{e}$; right panel: plasma frequency $f_{p}$. 
ly speaking, the results agree quite well with validation studies based on a comparison with F2-layer peak density and height data from mid-latitudes (Jakowski et al., 2004c). Significant differences do not primarily depend on the regional distance between the occultation point and the radar beam but can be attributed to ionospheric zonal and meridional small and large scale structures, which are enhanced during dawn and dusk and under perturbed geomagnetic conditions.

It is worth noting that the occultation technique apparently tends to overestimate the electron density in case of very low densities ( $c f$. fig. 7.5). A slight overestimation of the $F$ 2-layer peak density by radio occultation retrievals has been found also by validation studies based on midlatitude ionosonde data (cf. fig. 7.4; Jakowski et al., 2004c).

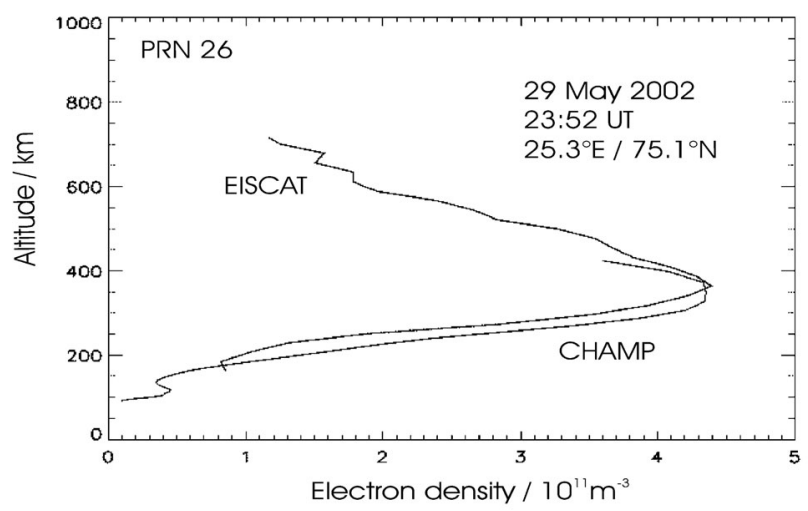

Fig. 7.5. Comparison of a CHAMP-IRO derived electron density profile with a corresponding EDP derived from EISCAT measurements on 29 May 2002, 23:52 UT.

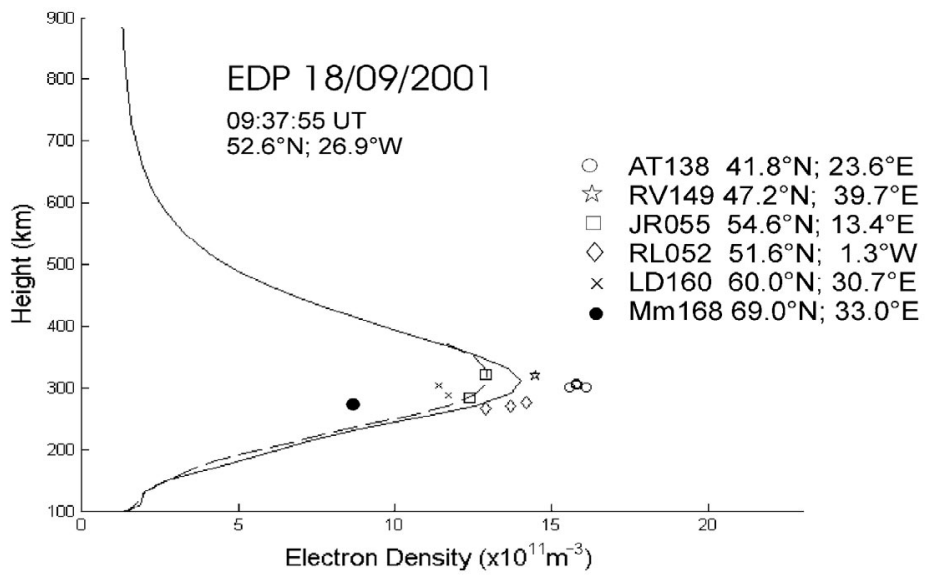

Fig. 7.6. Comparison of an IRO derived EDP (dashed line) with a corresponding profile extracted from the MIDAS reconstruction. Comparison with $N m F 2$ data from various European ionosonde stations is indicated by the symbols. 
Another option to evaluate the accuracy of the IRO derived EDPs is the comparison with the in situ electron density measurements performed by the planar Langmuir probe onboard CHAMP. The comparison of the Langmuir probe densities with the upper start values of the EDPs provides a quite consistent correlation (Jakowski et al., 2002a).

Beside this direct comparison with independent data sources a comparison with data driven reconstructions of the 4D electron density distribution is very promising. This has been done with reconstruction results of the MIDAS (Multi-Instrument Data Analysis System) algorithm (see also subsequent section). The MIDAS algorithm is designed to assimilate data from a number of different measurement techniques, thus allowing the spatial and temporal factors to be accounted for in the inversion. In the validation study (Spalla et al., 2002, 2003) various ionospheric data have been collected over Europe: GPS data from the International GPS Service network, true height profiles from vertical ionograms, and NIMS (TRANSIT) observations from Italy.

The experimental results indicate excellent agreement between the specification of ionospheric electron concentration using MIDAS and that calculated from CHAMP radio occultation ( $c f$. fig. 7.6).

\subsection{USE OF RO MEASUREMENTS FOR 3D RECONSTRUCTIONS}

\subsubsection{Background}

The last 15 years have seen a rapid growth in the development and use of ionospheric imaging systems. Traditional tomographic imaging techniques represent the ionosphere by means of a grid of pixels. Then, in the case of an integrated measurement, such as GPS-TEC, the $i$ th observation can be modelled by the sum of the electron density in the $j$ th pixel $\left(x_{j}\right)$ multiplied by the ray length within the $j$ th pixel $\left(H_{i j}\right)$ (fig. 7.7)

$$
y_{i}=\sum_{j=1}^{n} H_{i j} x_{j} H i j
$$

or, in matrix notation

$$
y=H x
$$

where $H$ is known as the observation operator, $x$ is the rasterised representation of the inosphere and $y$ is the TEC.

\subsubsection{Extension to 3D}

It can be seen that this formulation of the imaging problem is entirely general and can, therefore, be easily extended to a 3D grid of voxels. Furthermore, there is no requirement that the slant TEC must be measured by a ground station, and consequently it is also simple to directly include measurements of TEC made in low earth orbit. This generalisation does require that the ray paths from the GPS to the LEO satellite remain straight. In practice, the departure of the rays from straight line propagation in the ionosphere can reach a few kilometres at worst, which is much smaller than the typical vertical scale height in the $F$-region ionosphere (Schreiner et al., 1999). Therefore, bending can be neglected.

One advantage of using IRO slant TEC directly in an imaging system is that these techniques show the potential to overcome the limitations of the traditional Abel transform. In particular there is no requirement to assume conditions of spherical symmetry; there is no requirement for the measurements to be in the LEO's orbital plane; and there is no requirement for the transmitter and receiv- 


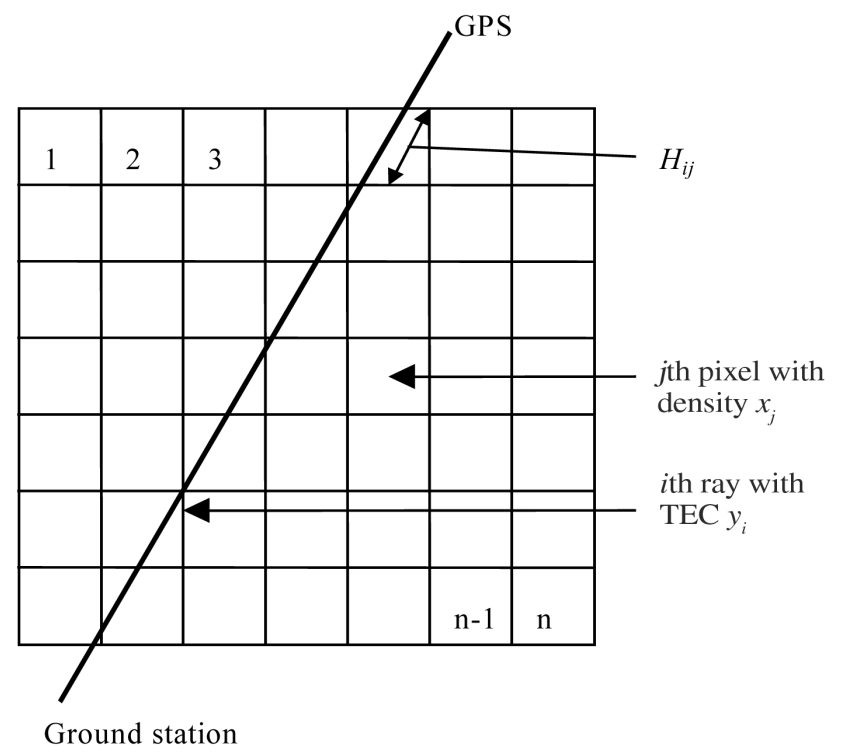

Fig. 7.7. Diagram illustrating the construction of the observation operator that relates a slant TEC measurement to a pixel representation of the ionosphere.

er to be in free space. Furthermore, the use of IRO slant TEC is complementary to the use of ground based measurements. This is first because IRO can provide largely horizontal ray paths in contrast to the largely vertical ray paths available from ground based GPS-TEC measurements; and secondly IRO can provide data over inaccessible areas of the world, such as the oceans.

The disadvantage of moving from 2D to 3D imaging is that a very large amount of data is required to be able to operate the traditional tomographical methods. In practice, and especially for GPS data (where the satellites are slow moving), the required quantity of data is not available, and other, more sophisticated, methods must be used. These methods must use a priori data to constrain the inversion from slant TEC to the electron density image. This a priori data may be a model of the ionosphere, or may take the form of assumptions about how the ionosphere can be decomposed into a set of basis functions.

\subsubsection{Example systems}

\subsubsection{MIDAS}

The Multi-Instrument Data Analysis System (MIDAS) was developed by the University of Bath to allow a range of data sources, including IRO, to be combined into an ionospheric image (Meggs et al., 2002; Mitchell and Spencer, 2002). The system is an extension of Discrete Inverse Theory (DIT) tomography (Fremouw and Secan, 1992; Fremouw et al., 1994). DIT provides a means of employing minimum variance techniques with an ionosphere described by ortho-normal basis and harmonic functions. MIDAS allows two, three and four dimensional inversions, where the latter includes the evolution of the ionospheric state with time. MIDAS differs from DIT in relying on singular value decomposition rather than a generalised inverse to perform the necessary matrix inversion steps of the algorithm. 


\subsubsection{EDAM}

Weighted, damped least mean squares estimation is a form of minimum variance optimal estimation (also referred to as Best Linear Unbiased Estimation - BLUE) that can provide an expression for an updated estimation of the ionospheric state (known as the analysis) that is dependent upon an initial estimate of the state (the background model), and the differences between the background model and the observations (Twomey, 1977; Menke, 1989). The error covariance matrices of the background model and the observations are also included to control the relative contributions of the background and the observations to the analysis

$$
\begin{gathered}
x_{a}=x_{b}+K\left(\boldsymbol{y}-H x_{b}\right) \\
K=B H^{T}\left(H B H^{T}+R\right)^{-1}
\end{gathered}
$$

where $x_{a}$ is the analysis, $x_{b}$ is the background model, $K$ is the weight matrix, $y$ is the observation vector, $B$ is the background error covariance matrix, and $R$ is the error covariance matrix of the observations (Bouttier and Courtier, 1999).

This technique has been employed in the Electron Density Assimilative Model (EDAM) developed by QinetiQ in the U.K. (Angling and Cannon, 2004). EDAM uses the Parameterised Ionospheric Model (PIM) as its background model and a background error covariance matrix that is also

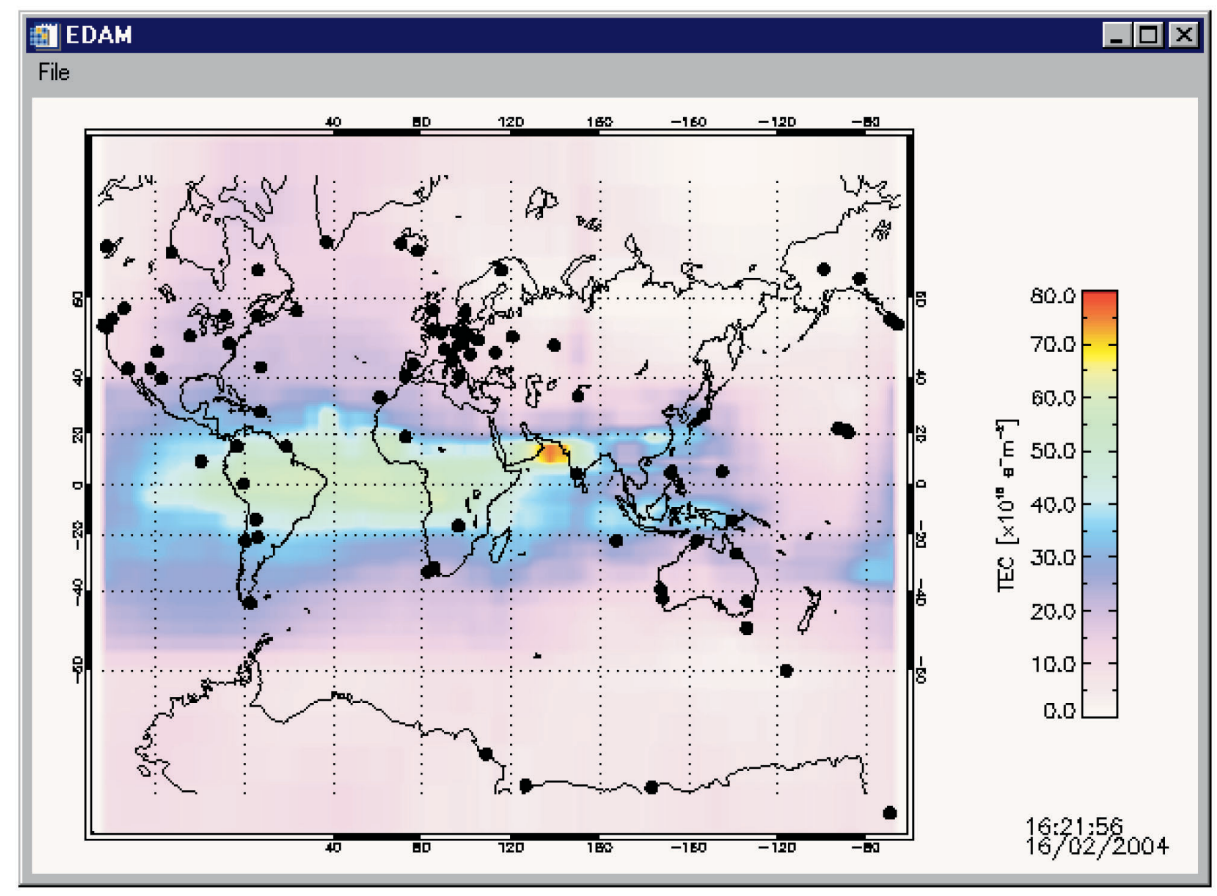

Fig. 7.8. Screen grab showing vertical TEC generated from the EDAM electron density analysis. The dots show the locations of contributing IGS stations. 
determined from the background model. EDAM has been tested using measurements simulated by ray tracing through ionospheric models and through tomographic images. It can also be run using data from the International GPS Service (IGS) and from the CHAMP satellite (Section 7.3).

\subsection{RO TECHNIQUE AS A FUTURE TOOL FOR IONOSPHERIC SOUNDING}

Onboard CHAMP the first ionospheric radio occultation measurements were carried out on 11 April 2001. Since that time we have obtained more than 80000 vertical electron density profiles which provide a huge data pool for ionospheric studies.

Although not yet completely validated, this tremendous data set already enables a preliminary data analysis to find out whether well-known physical ionospheric features are visible or not. One of the ionospheric key parameters is the peak electron density $N m F 2$ that is related to the ionospheric critical frequency $f 0 F 2$ by the relation $N m F 2=0.0124 \times(f 0 F 2)^{2}$ in SI units.

The strong latitudinal dependence of the ionospheric ionisation is clearly indicated in fig. 7.9 during night-time hours (18:00-06:00 LT) at all longitude sectors for northern summer conditions (days 121-222). The ionisation level is clearly enhanced under summer conditions in the northern hemisphere. It is worth noting that the day-time peak electron density is smaller in summer at midlatitudes. This so-called winter anomaly effect is not observed at night time when the ionisation is stored at greater heights. In the day-time the enhanced density of molecular constituents in summer leads to an enhanced plasma loss resulting in a low plasma density although the photo-ionisation rate is increased. Furthermore, fig. 7.9 illustrates quite well the effective maintenance of the night-time ionisation level at low latitudes as a result of the upward plasma drift due to the fountain effect around the geomagnetic equator during the day-time.The dispersion values are less than $50 \%$ of the original measurements except in the southern crest region.

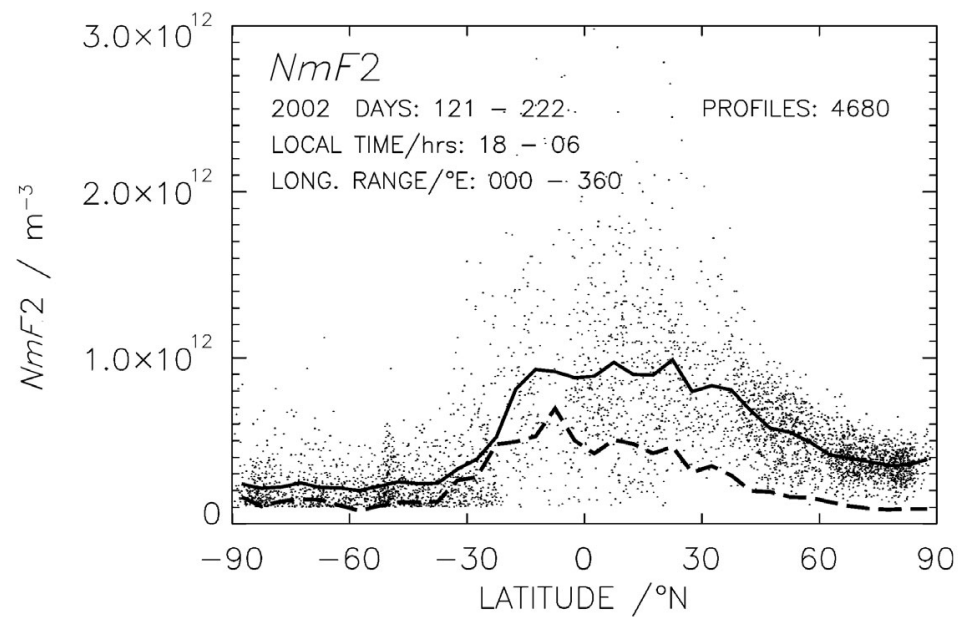

Fig. 7.9. Latitudinal distribution of $N m F 2$ for night-time hours (18-06 LT) during northern summer. The dashed curve represents the standard deviation. 
Because the summer hemisphere is characterized by a stronger energy input that leads not only to enhanced photo-ionisation but also to an enhanced heating of the thermosphere/ionosphere system, one should observe an expansion of the electron density profile shape that is accompanied by an uplifting of the $F$-layer peak density height $h m F 2$. Indeed, this behaviour is clearly shown in fig. 7.10 where the peak density height continuously grows from the winter towards the summer hemisphere. Due to the upward lifting of plasma in the low latitude range the corresponding peak density height is additionally enhanced.

Principally, it has to be mentioned that due to the rather low CHAMP orbit height of about $430 \mathrm{~km}$ the $h m F 2$ values are constrained to values less than about $400 \mathrm{~km}$. This causes a slight reduction in the average $h m F 2$ value. The standard deviation is usually less than $50 \mathrm{~km}$ except at high latitudes $\left(>70^{\circ}\right)$.

In addition to the bottomside slab thickness and $h m F 2$ the solar irradiance controlled expansion of the electron density profile is also visible in the topside scale height that is deduced at $425 \mathrm{~km}$ from the EDPs (fig. 7.11).

The higher plasma scale height at the summer hemisphere can be explained by enhanced plasma temperatures. In fact, this effect is also emphasized in the computed equivalent slab thickness $\tau_{b}=\mathrm{TEC}(h m F 2) / N m F 2$ of the bottom side electron density profile (fig. 7.12).

The scale height is just taken from the upper boundary region of the measurements where the topside model still has a certain influence. Hence, these data demonstrate clearly the adjustment of the scale height (start value $80 \mathrm{~km}$ ) during the initial phase of the retrieval procedure.

Although the IRO data reported here need more validation, a preliminary comparison with ionospheric 3D models may be helpful to get more knowledge about the data quality and to get some indication of the quality of the model. Following this idea, comparative studies were made with the IRI (Jakowski and Tsybulya, 2003) and with the NeQuick model already discussed in Section 7.2 (Jakowski et al., 2004b). In the subsequent comparison of IRO retrievals with NeQuick (Hochegger et al., 2000) we used more than 50000 profiles which have been provided by the automatic process-

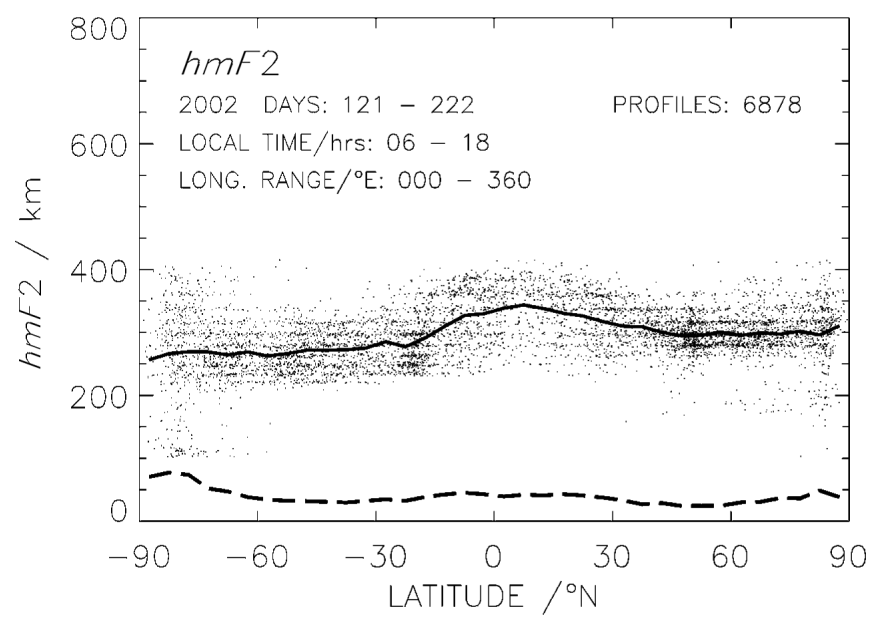

Fig. 7.10. Latitudinal distribution of the height of the peak density. The dashed curve represents the standard deviation. 


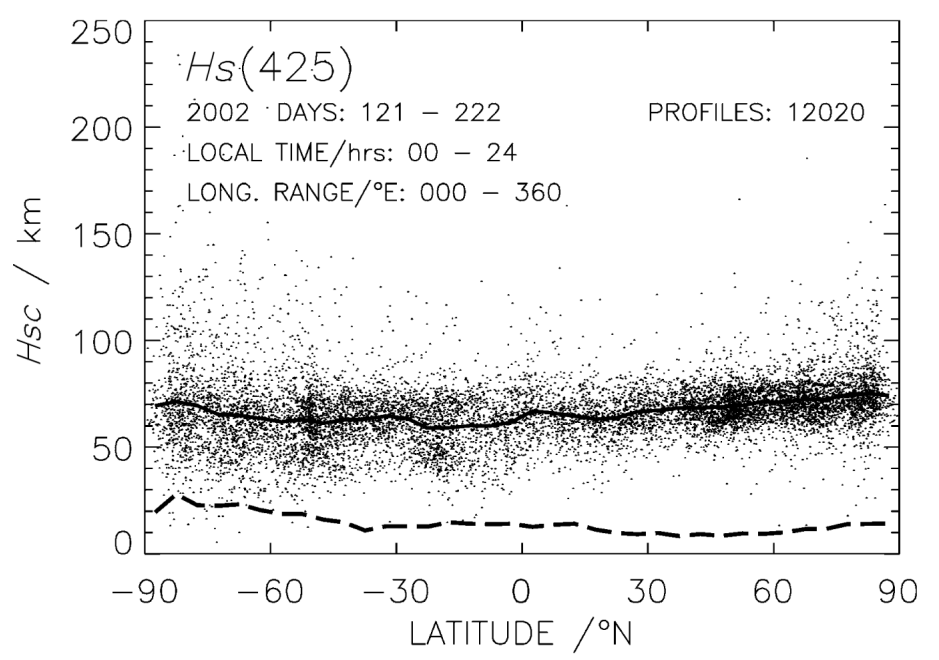

Fig. 7.11. Latitudinal distribution of the topside scale height taken at $425 \mathrm{~km}$ height at all longitude sectors over $24 \mathrm{~h}$. The dashed curve represents the standard deviation.

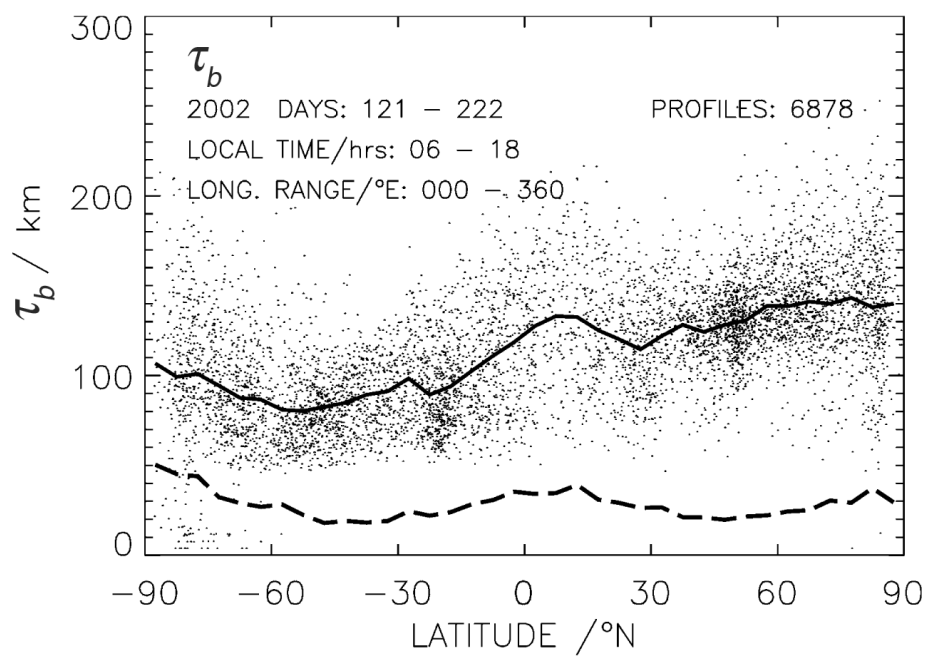

Fig. 7.12. Latitudinal distribution of the equivalent slab thickness of the bottomside ionosphere. The dashed curve represents the standard deviation.

ing system without additional checking. The comparison of IRO with corresponding NeQuick data indicates a systematic underestimation of the electron density in the $200-300 \mathrm{~km}$ altitude range of NeQuick compared with the IRO data (fig. 7.13).

Summarising this section it can be stated that IRO measurements have a big potential for studying the global ionosphere, and for developing and/or evaluating ionospheric models (e.g., Jakowski et al., 2004a). 

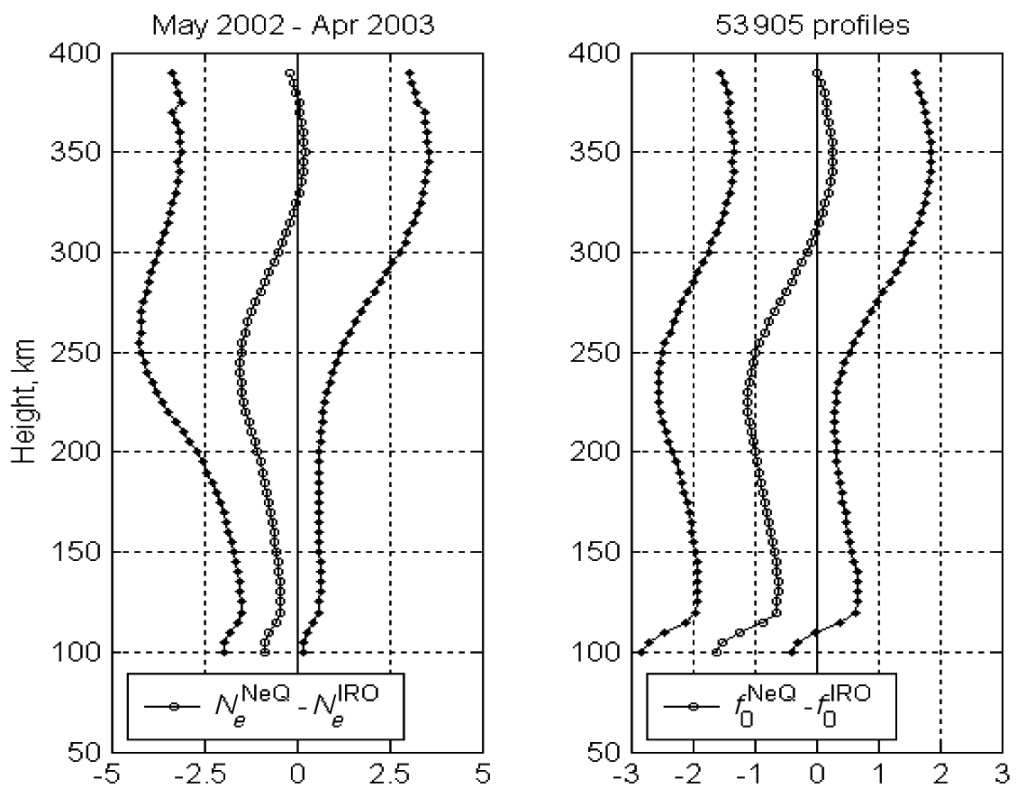

Deviation of electron density, $\Delta N_{e} / 10^{5} \mathrm{~cm}^{-3}$ Deviation of frequency, $\Delta$ f $\mathrm{MMH}$

Fig. 7.13. Statistical comparison of IRO and NeQuick derived EDPs. Left panel: electron density $N_{e}$; right panel: plasma frequency $f_{p}$.

\subsection{Summary AND CONClusions}

The GNSS radio occultation technique for measuring the vertical structure of the atmospheric refractivity from Low Earth Orbiting satellite (LEO) heights down to the Earth surface has been developed in recent years to a great extent on an international level. This innovative GNSS technique opens a new dimension for operational sounding of the ionosphere and atmosphere.

DLR has successfully inverted more than 80000 IRO measurements that provide a huge data pool for studying, modelling and monitoring the ionosphere. Although the validation work is not yet finished, ionospheric data products are already available via the ISDC Potsdam. Potential users should be aware of the principal average character of IRO derived electron density profiles. If compared with localized vertical sounding measurements the $F 2$-layer peak electron density $f 0 F 2$ and the corresponding height $h m F 2$ agree within about $17 \%$ and $13 \%$, respectively.

This agreement can certainly be improved, if additional information, e.g., on horizontal gradients or local densities, is included in the retrieval procedure. Horizontal gradients can be deduced from horizontal TEC maps such as those produced from ground based GPS measurements in DLR/IKN since 1995. An essential improvement is expected if IRO retrievals and tomographic reconstructions of the topside electron density distribution are combined to obtain a comprehensive view on the entire vertical electron density structure of the ionosphere from the bottomside up to GPS orbit heights.

The simultaneous inversion of both satellite-to-ground and satellite-to-satellite GPS data in a single algorithm provides a greatly improved geometry compared with individual data uses. The discussion of the early results has shown that the IRO retrieved electron density profiles and deduced parameters provide a consistent description of the general ionospheric behaviour. This fact indicates 
that IRO data should have great potential for studying a number of ionospheric phenomena on global scale in future. Furthermore, IRO measurements have great potential for developing and/or evaluating ionospheric models or for reconstructing the 3D electron density distribution of the ionosphere by data assimilation or tomographic techniques.

The planned launch of new occultation satellites over the next ten years offers great promise for modelling and monitoring the electron concentration in the near-Earth space for scientific studies and practical applications.

\section{ACKNOWLEDGEMENTS}

The work reported here was part of the COST 271/WG 2 activities. Thus the authors are grateful to the COST 271 members for numerous help by coordinated work during campaigns, valuable suggestions and critical comments. Particularly we thank the members of the CHAMP evaluation group of COST 271 for their coordinated activities. The authors are very grateful to all the colleagues from the international CHAMP team making available the use of GPS measurements performed onboard CHAMP.

\section{REFERENCES}

ANGLing, M.J. and P.S. CANNON (2004): Assimilation of radio occultation measurements into background ionospheric models, in Proceedings of the Ionospheric Effects Symposium, May 7-9, 2002, Alexandria, Virginia, Radio Sci. (accepted).

BoutTIER, F. and P. CourTiER (1999): Data assimilation concepts and methods, in ECMWF Training Course Notes, European Centre for Medium-range Weather Forecast.

Bullen, K.E. (1963): An Introduction to the Theory of Seismology (Cambridge University Press, Cambridge), 3rd edition.

Davies, K. (1990): Ionospheric Radio (Peter Peregrinus Ltd., London, U.K.).

Fremouw, E.J. and J.A. SECAN (1992): Application of stochastic inverse theory to ionospheric tomography, Radio Sci., 27 (5), 721-732.

Fremouw, E.J., J.A. SECAN, R.M. BuSSEY and B.M. Howe (1994): A status report on applying discreteinverse theory to ionospheric tomography, Int. J. Imaging Sys. Technol., 5, 97-105.

Garcia-Fernandez, M., A. Aragon, M. Hernandez-Pajares, J.M. Juan, J. Sanz, and V. Rios (2003): Ionospheric tomography with GPS data from CHAMP and SAC-C, in Proceedings of the Second CHAMP Science Meeting, 1-4 September 2003, GFZ Potsdam (accepted).

HAJJ, G.A. and L.J. Romans (1998): Ionospheric electron density profiles obtained with the Global Positioning System: results from the GPS/MET experiment, Radio Sci., 33, 175-190.

Heise, S., N. Jakowski, A. WehrenPfennig, Ch. Reigber and H. LüHr (2002): Sounding of the topside ionosphere/plasmasphere based on GPS measurements from CHAMP: initial results, Geophys. Res. Lett., 29 (14), 10.1029/2002GL014738.

Herglotz, G. (1907): Über das Benndorfsche Problem der Fortpflanzungsgeschwindigkeit der Erdbebenstrahlen, Phys. Z., 8, 145-147.

HochegGER, G. and R. LEITINGER (2000): Inversions of satellite to satellite electron content: simulation studies with NeUoG-plas, Phys. Chem. Earth (C), 25 (4), 353-357.

Hochegger, G. and R. LeITINGER (2001): Model assisted inversion of GNSS occultation data, Phys. Chem. Earth (C), 26 (5), 325-330.

Hochegger, G., B. NAva, S.M. Radicella and R. Leitinger (2000): A family of ionospheric models for different uses, Phys. Chem. Earth (C), 25 (4), 307-310.

JAKOWSKI, N. (1999): Capabilities of radio occultation measurements onboard LEO satellites for ionospheric monitoring and research, in Proceedings of the 4th COST 251 Workshop «The Impact 
of the Upper Atmosphere on Terrestrial and Earth-Space Communications», 22-25 March, Funchal, Madeira, Portugal, edited by A. VERnON, 116-121.

JAKOWSKI, N. and K. TSYBULYA (2003): Comparison of ionospheric radio occultation CHAMP data with IRI-2001, IRI Newsletter, 10 (1, 2, 4-7).

Jakowski, N., A. Wehrenpfennig, S. Heise, Ch. Reigber, H. LÜhr, L. GrunwaldT and T. Meehan (2002a): GPS Radio Occultation Measurements of the Ionosphere from CHAMP: Early Results, Geophys. Res. Lett., 29 (10), 10.1029/2001GL014364.

Jakowski, N., I. S. Kutiev, S. Heise and A. WehrenPFEnNig (2002b): A topside ionosphere/plasmasphere model for operational applications, on CD-Proceedings of the XXVIIth General Assembly of URSI, 17-24 August 2002, Maastricht, The Netherlands.

Jakowski, N., K. Tsybulya, S.M. Stankov and A. WehrenPFEnnig (2004a): About the potential of GPS radio occultation measurements for exploring the ionosphere, in Proceedings of the Second CHAMP Science Meeting, 1-4 September 2003, GFZ Potsdam (accepted).

Jakowski, N., K. Tsybulya, J. Mielich, A. Belehaki, D. Altadill, J.C. Jodogne and B. Zolesi, (2004b): Validation of GPS Radio Occultation Measurements on CHAMP by Vertical Sounding Observations in Europe, in Proceedings of the 2nd CHAMP Science Meeting, 1-4 September 2003, GFZ Potsdam (accepted).

JAKOWSKI, N., S. HeISE, A. WEHRENPFENNIG and K. TSYBUlYA (2004c): Ionospheric radio occultation measurements and space weather, in Proceedings of the OPAC-1 Meeting, 16-20 September 2002, Graz, Austria (accepted).

Jakowski, N., K. Tsybulya, S.M. Radicella, M. Cueto and M. Herraiz (2004d): Comparison of oonospheric electron density profiles from CHAMP Satellite with NeQuick model, in Proceedings of the 2nd CHAMP Science Meeting, 1-4 September 2003, GFZ Potsdam (accepted).

JefFreys, H. (1976): The Earth. Its Originam History and Physical Constitution (Cambridge University Press, Cambridge), 6th edition.

Kursinski, E.R., G.A. HAJJ, J.T. Schofield, R.P. Linfield and K.R. HARdY (1997): Observing Earth's atmosphere with radio occultation measurements using the Global Positioning System, $J$. Geophys. Res., 102 (D19), 23,429-23,465.

Meggs, R.W., C.N. Mitchell and P.S.J. Spencer (2002): Comparison of thin shell and 4D inversion techniques for measurement and mapping of total electron content using GPS signals over Europe, paper presented at Ionospheric Effects Symposium, Alexandria, Virginia, U.S.A.

Menke, W. (1989): Geophysical Data Analysis: Discrete Inverse Theory (International Geophysics Series, San Diego, Academic Press).

Mitchell, C.N. and P.S.J. SPENCER (2002): Development of tomographic techniques for large scale ionospheric imaging, paper presented at Ionospheric Effects Symposium, Alexandria, Virginia, U.S.A., 601-608.

O'Loughlin, K.F. (1997): SPIDR on the Web: Space Physics Interactive Data Resource on-line analysis tool, Radio Sci., 32, 2021-2026.

ReIGBer, CH., H. LÜHR and P. SCHWInTZER (2000): CHAMP mission status and perspectives, Eos, Trans. Am. Geophys. Un., 81 (suppl. to no. 48), F307.

SchreIner, W.S., S.V. Sokolovski and C. Rocken (1999): Analysis and validation of GPS/MET radio occultation data in the ionosphere, Radio Sci., 34, 949-966.

Spalla, P., N. Jakowski, C.N. Mitchell, P.S.J. Spencer and A. Wehrenpfennig (2002): Validation of CHAMP ionospheric radio-occultation observations over Europe, in Proceedings of the Ionospheric Effects Symposium, 7-9 May 2002, Alexandria, Virginia, 442-449.

Spalla, P., N. Jakowski, A. Wehrenpfennig, P.S. Spencer and C.N. Mitchell (2003): Verification of CHAMP radio occultation observations in the ionosphere using MIDAS, first CHAMP science mission results for gravity, in Magnetic and Atmospheric Studies, edited by CH. REIGBER, H. LÜHR and P. Schwintzer (Springer Verl., Berlin, Heidelberg-New York), 545-550.

STAMPER, R., J. LILENSTEN and N. JAKOWSKI (2004): Nowcasting, forecasting and warning for ionos- 
pheric propagation: supporting databases, Ann. Geophysics, 47 (suppl. to no. 2/3), 945-955 (this volume)

STANKOV, S.M. and N. JAKOWSKI (2004): Topside plasma scale height based on CHAMP measurements: first Results, in Proceedings of the Second CHAMP Science Meeting, 1-4 September 2003, GFZ Potsdam (accepted).

Stolle, C., N. JAKOwsKi, K. Schlegel and M. RietVeld (2004): Comparison of high latitude electron density profiles obtained with the GPS radio occultation technique and EISCAT measurements, Ann. Geophysicae, 22 (6), 2015-2028.

Twomey, S. (1977): Introduction to the Mathematics of Inversion in Remote Sensing and Indirect Measurements (New York, Dover Publications).

WeHrenPFEnNig, A., N. JAKOWSKI and J. WiCKERT (2001): A dynamically configurable system for operational processing of space weather data, Phys. Chem. Earth, 26, 601-604.

Wickert, J., G. Beyerle, G.A. HAJJ, V. Schwieger and Ch. ReIgber (2002): GPS radio occultation with CHAMP: Atmospheric profiling utilizing the space-based single difference technique, Geophys. Res. Lett., 29 (8), 10.1029/2001GL013982.

WiEChERT, E. and L. Geiger (1910): Bestimmung des Weges der Erdbebenwellen im Erdinnern, Phys. Z., 11, 294-312. 